\title{
Monotone Operators without Enlargements
}

\author{
Jonathan M. Borwein, Regina Burachikł and Liangjin Yao
}

October 12, 2011

\begin{abstract}
Enlargements have proven to be useful tools for studying maximally monotone mappings. It is therefore natural to ask in which cases the enlargement does not change the original mapping. Svaiter has recently characterized non-enlargeable operators in reflexive Banach spaces and has also given some partial results in the nonreflexive case. In the present paper, we provide another characterization of non-enlargeable operators in nonreflexive Banach spaces under a closedness assumption on the graph. Furthermore, and still for general Banach spaces, we present a new proof of the maximality of the sum of two maximally monotone linear relations. We also present a new proof of the maximality of the sum of a maximally monotone linear relation and a normal cone operator when the domain of the linear relation intersects the interior of the domain of the normal cone.
\end{abstract}

\section{Mathematics Subject Classification:}

Primary 47A06, 47H05; Secondary 47B65, 47N10, 90C25

Keywords: Adjoint, Fenchel conjugate, Fitzpatrick function, linear relation, maximally monotone operator, monotone operator, multifunction, normal cone operator, nonenlargeable operator, operator of type (FPV), partial inf-convolution, set-valued operator.

\footnotetext{
${ }^{*}$ CARMA, University of Newcastle, Newcastle, New South Wales 2308, Australia. E-mail: jonathan.borwein@newcastle.edu.au. Distinguished Professor King Abdulaziz University, Jeddah.

†School of Mathematics and Statistics, University of South Australia, Mawson Lakes, SA 5095, Australia. E-mail: regina.burachik@unisa.edu.au.

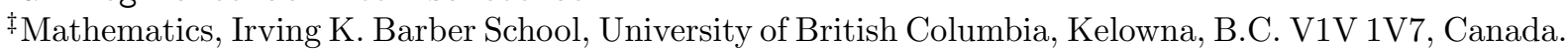
E-mail: $1 j$ inyao@interchange.ubc.ca.
} 


\section{Introduction}

Maximally monotone operators have proven to be a significant class of objects in both modern Optimization and Functional Analysis. They extend both the concept of subdifferentials of convex functions, as well as that of a positive semi-definite function. Their study in the context of Banach spaces, and in particular nonreflexive ones, arises naturally in the theory of partial differential equations, equilibrium problems, and variational inequalities. For a detailed study of these operators, see, e.g., [12, 13, 14, or the books [6, 15, 19, 25, 31, 32, 30, 41, 42.

A useful tool for studying or proving properties of a maximally monotone operator $A$ is the concept of the "enlargement of $A$ ". A main example of this usefulness is Rockafellar's proof of maximality of the subdifferential of a convex function (Fact 3.3 below), which uses the concept of $\varepsilon$-subdifferential. The latter is an enlargement of the subdifferential introduced in [17].

Broadly speaking, an enlargement is a multifunction which approximates the original maximally monotone operator in a convenient way. Another useful way to study a maximally monotone operator is by associating to it a convex function called the Fitzpatrick function. The latter was introduced by Fitzpatrick in [21] and its connection with enlargements, as shown in [20], is contained in (4) below.

Our first aim in the present paper is to provide further characterizations of maximally monotone operators which are not enlargeable, in the setting of possibly nonreflexive Banach spaces (see Section 4). In other words, in which cases the enlargement does not change the graph of a maximally monotone mapping defined in a Banach space? We address this issue Corollary 4.2, under a closedness assumption on the graph of the operator.

Our other aim is to use the Fitzpatrick function to derive new results which establish the maximality of the sum of two maximally monotone operators in nonreflexive spaces (see Section 5). First, we provide a different proof of the maximality of the sum of two maximally monotone linear relations. Second, we provide a proof of the maximality of the sum of a maximally monotone linear relation and a normal cone operator when the domain of the operator intersects the interior of the domain of the normal cone.

\section{Technical Preliminaries}

Throughout this paper, $X$ is a real Banach space with norm $\|\cdot\|$, and $X^{*}$ is the continuous dual of $X$. The spaces $X$ and $X^{*}$ are paired by the duality pairing, denoted as $\langle\cdot, \cdot\rangle$. The space $X$ is identified with its canonical image in the bidual space $X^{* *}$. Furthermore, $X \times X^{*}$ 
and $\left(X \times X^{*}\right)^{*}:=X^{*} \times X^{* *}$ are paired via $\left\langle\left(x, x^{*}\right),\left(y^{*}, y^{* *}\right)\right\rangle:=\left\langle x, y^{*}\right\rangle+\left\langle x^{*}, y^{* *}\right\rangle$, where $\left(x, x^{*}\right) \in X \times X^{*}$ and $\left(y^{*}, y^{* *}\right) \in X^{*} \times X^{* *}$.

Let $A: X \rightrightarrows X^{*}$ be a set-valued operator (also known as a multifunction) from $X$ to $X^{*}$, i.e., for every $x \in X, A x \subseteq X^{*}$, and let gra $A:=\left\{\left(x, x^{*}\right) \in X \times X^{*} \mid x^{*} \in A x\right\}$ be the graph of $A$. The domain of $A$ is $\operatorname{dom} A:=\{x \in X \mid A x \neq \varnothing\}$, and $\operatorname{ran} A:=A(X)$ for the range of $A$. Recall that $A$ is monotone if

$$
\left\langle x-y, x^{*}-y^{*}\right\rangle \geq 0, \quad \forall\left(x, x^{*}\right) \in \operatorname{gra} A \forall\left(y, y^{*}\right) \in \operatorname{gra} A,
$$

and maximally monotone if $A$ is monotone and $A$ has no proper monotone extension (in the sense of graph inclusion). Let $A: X \rightrightarrows X^{*}$ be monotone and $\left(x, x^{*}\right) \in X \times X^{*}$. We say $\left(x, x^{*}\right)$ is monotonically related to gra $A$ if

$$
\left\langle x-y, x^{*}-y^{*}\right\rangle \geq 0, \quad \forall\left(y, y^{*}\right) \in \operatorname{gra} A .
$$

Let $A: X \rightrightarrows X^{*}$ be maximally monotone. We say $A$ is of type (FPV) if for every open convex set $U \subseteq X$ such that $U \cap \operatorname{dom} A \neq \varnothing$, the implication

$$
x \in U \text { and }\left(x, x^{*}\right) \text { is monotonically related to gra } A \cap U \times X^{*} \Rightarrow\left(x, x^{*}\right) \in \operatorname{gra} A
$$

holds. Maximally monotone operators of type (FPV) are relevant primarily in the context of nonreflexive Banach spaces. Indeed, it follows from [32, Theorem 44.1] and a well-known result from [28] that every maximally monotone operator defined in a reflexive Banach space is of type (FPV). As mentioned in [32, §44], an example of a maximally monotone operator which is not of type (FPV) has not been found yet.

Let $A: X \rightrightarrows X^{*}$ be monotone such that gra $A \neq \varnothing$. The Fitzpatrick function associated with $A$ is defined by

$$
\left.\left.F_{A}: X \times X^{*} \rightarrow\right]-\infty,+\infty\right]:\left(x, x^{*}\right) \mapsto \sup _{\left(a, a^{*}\right) \in \operatorname{gra} A}\left(\left\langle x, a^{*}\right\rangle+\left\langle a, x^{*}\right\rangle-\left\langle a, a^{*}\right\rangle\right) .
$$

When $A$ is maximally monotone, a fundamental property of the Fitzpatrick function $F_{A}$ (see Fact 3.5) is that

$$
\begin{aligned}
& F_{A}\left(x, x^{*}\right) \geq\left\langle x, x^{*}\right\rangle \text { for all }\left(x, x^{*}\right) \in X \times X^{*}, \\
& F_{A}\left(x, x^{*}\right)=\left\langle x, x^{*}\right\rangle \text { for all }\left(x, x^{*}\right) \in \operatorname{gra} A .
\end{aligned}
$$

Hence, for a fixed $\varepsilon \geq 0$, the set of pairs $\left(x, x^{*}\right)$ for which $F_{A}\left(x, x^{*}\right) \leq\left\langle x, x^{*}\right\rangle+\varepsilon$ contains the graph of $A$. This motivates the definition of enlargement of $A$ for a general monotone mapping $A$, which is as follows.

Let $\varepsilon \geq 0$. We define $A_{\varepsilon}: X \rightrightarrows X^{*}$ by

$$
\begin{aligned}
\operatorname{gra} A_{\varepsilon} & :=\left\{\left(x, x^{*}\right) \in X \times X^{*} \mid\left\langle x^{*}-y^{*}, x-y\right\rangle \geq-\varepsilon, \forall\left(y, y^{*}\right) \in \operatorname{gra} A\right\} \\
& =\left\{\left(x, x^{*}\right) \in X \times X^{*} \mid F_{A}\left(x, x^{*}\right) \leq\left\langle x, x^{*}\right\rangle+\varepsilon\right\} .
\end{aligned}
$$


Let $A: X \rightrightarrows X^{*}$ be monotone. We say $A$ is enlargeable if gra $A \varsubsetneqq \operatorname{gra} A_{\varepsilon}$ for some $\varepsilon \geq 0$, and $A$ is non-enlargeable if gra $A=\operatorname{gra} A_{\varepsilon}$ for every $\varepsilon \geq 0$. Lemma 23.1 in 32 proves that if a proper and convex function verifies (2), then the set of all pairs $\left(x, x^{*}\right)$ at which (3) holds is a monotone set. Therefore, if $A$ is non-enlargeable then it must be maximally monotone.

We adopt the notation used in the books [15, Chapter 2] and [12, 31, 32]. Given a subset $C$ of $X$, int $C$ is the interior of $C, \bar{C}$ is the norm closure of $C$. The support function of $C$, written as $\sigma_{C}$, is defined by $\sigma_{C}\left(x^{*}\right):=\sup _{c \in C}\left\langle c, x^{*}\right\rangle$. The indicator function of $C$, written as $\iota_{C}$, is defined at $x \in X$ by

$$
\iota_{C}(x):= \begin{cases}0, & \text { if } x \in C ; \\ +\infty, & \text { otherwise }\end{cases}
$$

For every $x \in X$, the normal cone operator of $C$ at $x$ is defined by $N_{C}(x):=\left\{x^{*} \in X^{*} \mid\right.$ $\left.\sup _{c \in C}\left\langle c-x, x^{*}\right\rangle \leq 0\right\}$, if $x \in C$; and $N_{C}(x):=\varnothing$, if $x \notin C$. The closed unit ball is $B_{X}:=\{x \in X \mid\|x\| \leq 1\}$, and $\mathbb{N}:=\{1,2,3, \ldots\}$.

If $Z$ is a real Banach space with dual $Z^{*}$ and a set $S \subseteq Z$, we denote $S^{\perp}$ by $S^{\perp}:=\left\{z^{*} \in\right.$ $\left.Z^{*} \mid\left\langle z^{*}, s\right\rangle=0, \quad \forall s \in S\right\}$. The adjoint of an operator $A$, written $A^{*}$, is defined by

$$
\operatorname{gra} A^{*}:=\left\{\left(x^{* *}, x^{*}\right) \in X^{* *} \times X^{*} \mid\left(x^{*},-x^{* *}\right) \in(\operatorname{gra} A)^{\perp}\right\} .
$$

We will be interested in monotone operators which are linear relations, i.e., such that gra $A$ is a linear subspace. Note that in this situation, $A^{*}$ is also a linear relation. Moreover, $A$ is symmetric if gra $A \subseteq$ gra $A^{*}$. Equivalently, for all $\left(x, x^{*}\right),\left(y, y^{*}\right) \in \operatorname{gra} A$ it holds that

$$
\left\langle x, y^{*}\right\rangle=\left\langle y, x^{*}\right\rangle \text {. }
$$

We say that a linear relation $A$ is skew if gra $A \subseteq \operatorname{gra}\left(-A^{*}\right)$. Equivalently, for all $\left(x, x^{*}\right) \in$ gra $A$ we have

$$
\left\langle x, x^{*}\right\rangle=0 .
$$

We define the symmetric part a of $A$ via

$$
A_{+}:=\frac{1}{2} A+\frac{1}{2} A^{*}
$$

It is easy to check that $A_{+}$is symmetric.

Let $f: X \rightarrow]-\infty,+\infty]$. Then $\operatorname{dom} f:=f^{-1}(\mathbb{R})$ is the domain of $f$, and $f^{*}: X^{*} \rightarrow$ $[-\infty,+\infty]: x^{*} \mapsto \sup _{x \in X}\left(\left\langle x, x^{*}\right\rangle-f(x)\right)$ is the Fenchel conjugate of $f$. We denote by $\bar{f}$ the lower semicontinuous hull of $f$. We say that $f$ is proper if $\operatorname{dom} f \neq \varnothing$. Let $f$ be proper. The subdifferential of $f$ is defined by

$$
\partial f: X \rightrightarrows X^{*}: x \mapsto\left\{x^{*} \in X^{*} \mid(\forall y \in X)\left\langle y-x, x^{*}\right\rangle+f(x) \leq f(y)\right\} .
$$


For $\varepsilon \geq 0$, the $\varepsilon$-subdifferential of $f$ is defined by

$$
\partial_{\varepsilon} f: X \rightrightarrows X^{*}: x \mapsto\left\{x^{*} \in X^{*} \mid(\forall y \in X)\left\langle y-x, x^{*}\right\rangle+f(x) \leq f(y)+\varepsilon\right\} .
$$

Note that $\partial f=\partial_{0} f$.

Relatedly, we say $A$ is of Brønsted-Rockafellar (BR) type [32, 15] if whenever $\left(x, x^{*}\right) \in$ $X \times X^{*}, \alpha, \beta>0$ while

$$
\inf _{\left(a, a^{*}\right) \in \operatorname{gra} A}\left\langle x-a, x^{*}-a^{*}\right\rangle>-\alpha \beta
$$

then there exists $\left(b, b^{*}\right) \in \operatorname{gra} A$ such that $\|x-b\|<\alpha,\left\|x^{*}-b^{*}\right\|<\beta$. The name is motivated by the celebrated theorem of Brønsted and Rockafellar [32, 15] which can be stated now as saying that all closed convex subgradients are of type (BR).

Let $g: X \rightarrow]-\infty,+\infty]$. The inf-convolution of $f$ and $g, f \square g$, is defined by

$$
f \square g: x \rightarrow \inf _{y \in X}[f(y)+g(x-y)] .
$$

Let $Y$ be another real Banach space. We set $P_{X}: X \times Y \rightarrow X:(x, y) \mapsto x$. We denote Id $: X \rightarrow X$ by the identity mapping.

Let $\left.\left.F_{1}, F_{2}: X \times Y \rightarrow\right]-\infty,+\infty\right]$. Then the partial inf-convolution $F_{1} \square_{2} F_{2}$ is the function defined on $X \times Y$ by

$$
F_{1} \square_{2} F_{2}:(x, y) \mapsto \inf _{v \in Y}\left[F_{1}(x, y-v)+F_{2}(x, v)\right]
$$

\section{$3 \quad$ Auxiliary results}

We collect in this section some facts we will use later on. These facts involve convex functions, maximally monotone operators and Fitzpatrick functions.

Fact 3.1 (See [25, Proposition 3.3 and Proposition 1.11].) Let $f: X \rightarrow]-\infty,+\infty$ ] be $a$ lower semicontinuous convex and int dom $f \neq \varnothing$. Then $f$ is continuous on int dom $f$ and $\partial f(x) \neq \varnothing$ for every $x \in \operatorname{int} \operatorname{dom} f$.

Fact 3.2 (Rockafellar) (See [27, Theorem 3(a)], [32, Corollary 10.3 and Theorem 18.1], or [41, Theorem 2.8.7(iii)].) Let $f, g: X \rightarrow]-\infty,+\infty$ ] be proper convex functions. Assume that there exists a point $x_{0} \in \operatorname{dom} f \cap \operatorname{dom} g$ such that $g$ is continuous at $x_{0}$. Then for every $z^{*} \in X^{*}$, there exists $y^{*} \in X^{*}$ such that

$$
(f+g)^{*}\left(z^{*}\right)=f^{*}\left(y^{*}\right)+g^{*}\left(z^{*}-y^{*}\right) .
$$


Fact 3.3 (Rockafellar) (See [29, Theorem A], [41, Theorem 3.2.8], [32, Theorem 18.7] or [23, Theorem 2.1]) Let $f: X \rightarrow]-\infty,+\infty$ ] be a proper lower semicontinuous convex function. Then $\partial f$ is maximally monotone.

Fact 3.4 (Attouch-Brézis) (See [1, Theorem 1.1] or [32, Remark 15.2]). Let f, $g: X \rightarrow$ ]$-\infty,+\infty]$ be proper lower semicontinuous and convex. Assume that $\bigcup_{\lambda>0} \lambda[\operatorname{dom} f-\operatorname{dom} g]$ is a closed subspace of $X$. Then

$$
(f+g)^{*}\left(z^{*}\right)=\min _{y^{*} \in X^{*}}\left[f^{*}\left(y^{*}\right)+g^{*}\left(z^{*}-y^{*}\right)\right], \quad \forall z^{*} \in X^{*} .
$$

Fact 3.3 above relates a convex function with maximal monotonicity. Fitzpatrick functions go in the opposite way: from maximally monotone operators to convex functions.

Fact 3.5 (Fitzpatrick) (See [21, Corollary 3.9] and [12, 15].) Let $A: X \rightrightarrows X^{*}$ be maximally monotone. Then for every $\left(x, x^{*}\right) \in X \times X^{*}$, the inequality $\left\langle x, x^{*}\right\rangle \leq F_{A}\left(x, x^{*}\right)$ is true, and the equality holds if and only if $\left(x, x^{*}\right) \in$ gra $A$.

It was pointed out in [32, Problem 31.3] that it is unknown whether $\overline{\operatorname{dom} A}$ is necessarily convex when $A$ is maximally monotone and $X$ is not reflexive. When $A$ is of type (FPV), the question was answered positively by using $F_{A}$.

Fact 3.6 (Simons) (See 32, Theorem 44.2].) Let $A: X \rightrightarrows X^{*}$ be of type (FPV). Then $\overline{\operatorname{dom} A}=\overline{P_{X}\left[\operatorname{dom} F_{A}\right]}$ and $\overline{\operatorname{dom} A}$ is convex.

We observe that when $A$ is of type (FPV) then also dom $A_{\varepsilon}$ has convex closure.

Remark 3.7 Let $A$ be of type (FPV) and fix $\varepsilon \geq 0$. Then by (4), Fact 3.5 and Fact 3.6, we have $\operatorname{dom} A \subseteq \operatorname{dom} A_{\varepsilon} \subseteq P_{X}\left[\operatorname{dom} F_{A}\right] \subseteq \overline{\operatorname{dom} A}$. Thus we obtain

$$
\overline{\operatorname{dom} A}=\overline{\left[\operatorname{dom} A_{\varepsilon}\right]}=\overline{P_{X}\left[\operatorname{dom} F_{A}\right]},
$$

and this set is convex because $\operatorname{dom} F_{A}$ is convex. As a result, for every $A$ of type (FPV) it holds that $\overline{\operatorname{dom} A}=\overline{\left[\operatorname{dom} A_{\varepsilon}\right]}$ and this set is convex.

We recall below some necessary conditions for a maximally monotone operator to be of type (FPV).

Fact 3.8 (Simons) (See [32, Theorem 46.1].) Let $A: X \rightrightarrows X^{*}$ be a maximally monotone linear relation. Then $A$ is of type (FPV).

Fact 3.9 (Fitzpatrick-Phelps and Verona-Verona) (See [22, Corollary 3.4], [36, Theorem 3] or [32, Theorem 48.4(d)].) Let $f: X \rightarrow]-\infty,+\infty$ ] be proper, lower semicontinuous, and convex. Then $\partial f$ is of type (FPV). 
Fact 3.10 (See [40, Corollary 3.3].) Let $A: X \rightrightarrows X^{*}$ be a maximally monotone linear relation, and $f: X \rightarrow]-\infty,+\infty]$ be a proper lower semicontinuous convex function with $\operatorname{dom} A \cap \operatorname{int} \operatorname{dom} \partial f \neq \varnothing$. Then $A+\partial f$ is of type $(F P V)$.

Fact 3.11 (Phelps-Simons) (See [26, Corollary 2.6 and Proposition 3.2(h)].) Let $A: X \rightarrow$ $X^{*}$ be monotone and linear. Then $A$ is maximally monotone and continuous.

Fact 3.12 (See [10, Theorem 4.2] or [24, Lemma 1.5].) Let $A: X \rightrightarrows X^{*}$ be maximally monotone such that gra $A$ is convex. Then gra $A$ is affine.

Fact 3.13 (Simons) (See [32, Lemma 19.7 and Section 22].) Let $A: X \rightrightarrows X^{*}$ be a monotone operator such that gra $A$ is convex with gra $A \neq \varnothing$. Then the function

$$
\left.\left.g: X \times X^{*} \rightarrow\right]-\infty,+\infty\right]:\left(x, x^{*}\right) \mapsto\left\langle x, x^{*}\right\rangle+\iota_{\operatorname{gra} A}\left(x, x^{*}\right)
$$

is proper and convex.

Fact 3.14 (See [38, Theorem 3.4 and Corollary 5.6], or [32, Theorem 24.1(b)].) Let A, B : $X \rightrightarrows X^{*}$ be maximally monotone operators. Assume that $\bigcup_{\lambda>0} \lambda\left[P_{X}\left(\operatorname{dom} F_{A}\right)-P_{X}\left(\operatorname{dom} F_{B}\right)\right]$ is a closed subspace. If

$$
F_{A+B} \geq\langle\cdot, \cdot\rangle \text { on } \quad X \times X^{*},
$$

then $A+B$ is maximally monotone.

Definition 3.15 (Fitzpatrick family) Let $A: X \rightrightarrows X^{*}$ be maximally monotone. The associated Fitzpatrick family $\mathcal{F}_{A}$ consists of all functions $\left.\left.F: X \times X^{*} \rightarrow\right]-\infty,+\infty\right]$ that are lower semicontinuous and convex, and that satisfy $F \geq\langle\cdot, \cdot\rangle$, and $F=\langle\cdot, \cdot\rangle$ on gra $A$.

Fact 3.16 (Fitzpatrick) (See [21, Theorem 3.10] or [20].) Let $A: X \rightrightarrows X^{*}$ be maximally monotone. Then for every $\left(x, x^{*}\right) \in X \times X^{*}$,

$$
F_{A}\left(x, x^{*}\right)=\min \left\{F\left(x, x^{*}\right) \mid F \in \mathcal{F}_{A}\right\} .
$$

Corollary 3.17 Let $A: X \rightrightarrows X^{*}$ be a maximally monotone operator such that gra $A$ is convex. Then for every $\left(x, x^{*}\right) \in X \times X^{*}$,

$$
F_{A}\left(x, x^{*}\right)=\min \left\{F\left(x, x^{*}\right) \mid F \in \mathcal{F}_{A}\right\} \quad \text { and } g\left(x, x^{*}\right)=\max \left\{F\left(x, x^{*}\right) \mid F \in \mathcal{F}_{A}\right\},
$$

where $g:=\langle\cdot, \cdot\rangle+\iota_{\operatorname{gra} A}$.

Proof. Apply Fact 3.13 and Fact 3.16,

Fact 3.18 (See [32, Lemma 23.9], or [7, Proposition 4.2].) Let $A, B: X \rightrightarrows X^{*}$ be monotone operators and $\operatorname{dom} A \cap \operatorname{dom} B \neq \varnothing$. Then $F_{A+B} \leq F_{A} \square_{2} F_{B}$. 
Let $X, Y$ be two real Banach spaces and let $h: X \times Y \rightarrow]-\infty,+\infty]$ be a convex function. We say that $h$ is separable if there exist convex functions $\left.\left.h_{1}: X \rightarrow\right]-\infty,+\infty\right]$ and $\left.\left.h_{2}: Y \rightarrow\right]-\infty,+\infty\right]$ such that $h(x, y)=h_{1}(x)+h_{2}(y)$. This situation is denoted as $h=h_{1} \oplus h_{2}$. We recall below some cases in which the Fitzpatrick function is separable.

Fact 3.19 (See [2, Corollary 5.9] or [5, Fact 4.1].) Let $C$ be a nonempty closed convex subset of $X$. Then $F_{N_{C}}=\iota_{C} \oplus \iota_{C}^{*}$.

Fact 3.20 (See [2, Theorem 5.3].) Let $f: X \rightarrow]-\infty,+\infty]$ be a proper lower semicontinuous sublinear function. Then $F_{\partial f}=f \oplus f^{*}$ and $\mathcal{F}_{A}=\left\{f \oplus f^{*}\right\}$.

Remark 3.21 Let $f$ be as in Fact 3.20 , then

$$
\begin{aligned}
\operatorname{gra}(\partial f)_{\varepsilon} & =\left\{\left(x, x^{*}\right) \in X \times X^{*} \mid f(x)+f^{*}\left(x^{*}\right) \leq\left\langle x, x^{*}\right\rangle+\varepsilon\right\} \\
& =\operatorname{gra} \partial_{\varepsilon} f, \quad \forall \varepsilon \geq 0 .
\end{aligned}
$$

Fact 3.22 (Svaiter) (See [35, page 312].) Let $A: X \rightrightarrows X^{*}$ be maximally monotone. Then $A$ is non-enlargeable if and only if gra $A=\operatorname{dom} F_{A}$ and then gra $A$ is convex.

It is immediate from the definitions that:

Fact 3.23 Every non-enlargeable maximally monotone operator is of type (BR).

Fact 3.20 and the subsequent remark refers to a case in which all enlargements of $A$ coincide, or, equivalently, the Fitzpatrick family is a singleton. It is natural to deduce that a non-enlargeable operator will also have a single element in its Fitzpatrick family.

Corollary 3.24 Let $A$ : $X \rightrightarrows X^{*}$ be maximally monotone. Then $A$ is non-enlargeable if and only if $F_{A}=\iota_{\text {gra } A}+\langle\cdot, \cdot\rangle$ and hence $\mathcal{F}_{A}=\left\{\iota_{\text {gra } A}+\langle\cdot, \cdot\rangle\right\}$.

Proof. " $\Rightarrow$ ": By Fact [3.22, we have gra $A$ is convex. By Fact 3.5 and Fact [3.22, we have $F_{A}=\iota_{\text {gra } A}+\langle\cdot, \cdot\rangle$. Then by Corollary $\left[3.17, \mathcal{F}_{A}=\left\{\iota_{\text {gra } A}+\langle\cdot, \cdot\rangle\right\}\right.$. " $\Leftarrow "$ : Apply directly Fact 3.22 .

Remark 3.25 The condition that $\mathcal{F}_{A}$ is singleton does not guarantee that gra $A$ is convex. For example, let $f: X \rightarrow]-\infty,+\infty]$ be a proper lower semicontinuous sublinear function. Then by Fact $3.20, \mathcal{F}_{A}$ is singleton but gra $\partial f$ is not necessarily convex.

\section{Non-Enlargeable Monotone Linear Relations}

We begin with a basic characterization: 
Theorem 4.1 Let $A: X \rightrightarrows X^{*}$ be a maximally monotone linear relation such that gra $A$ is

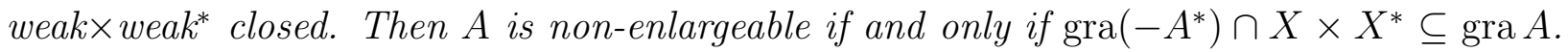
In this situation, we have that $\left\langle x, x^{*}\right\rangle=0, \forall\left(x, x^{*}\right) \in \operatorname{gra}\left(-A^{*}\right) \cap X \times X^{*}$.

Proof. " $\Rightarrow$ ": By Corollary 3.24,

$$
F_{A}=\iota_{\text {gra } A}+\langle\cdot, \cdot\rangle .
$$

Let $\left(x, x^{*}\right) \in \operatorname{gra}\left(-A^{*}\right) \cap X \times X^{*}$. Then we have

$$
\begin{aligned}
F_{A}\left(x, x^{*}\right) & =\sup _{\left(a, a^{*}\right) \in \operatorname{gra} A}\left\{\left\langle a^{*}, x\right\rangle+\left\langle a, x^{*}\right\rangle-\left\langle a, a^{*}\right\rangle\right\} \\
& =\sup _{\left(a, a^{*}\right) \in \operatorname{gra} A}\left\{-\left\langle a, a^{*}\right\rangle\right\} \\
& =0 .
\end{aligned}
$$

Then by (15), $\left(x, x^{*}\right) \in \operatorname{gra} A$ and $\left\langle x, x^{*}\right\rangle=0$. Hence $\operatorname{gra}\left(-A^{*}\right) \cap X \times X^{*} \subseteq \operatorname{gra} A$.

" $\Leftarrow$ ": By the assumption that gra $A$ is weak $\times$ weak* closed, we have

$$
\left[\operatorname{gra}\left(-A^{*}\right) \cap X \times X^{*}\right]^{\perp} \cap X^{*} \times X=\left[\left(\operatorname{gra} A^{-1}\right)^{\perp} \cap X \times X^{*}\right]^{\perp} \cap X^{*} \times X=\operatorname{gra} A^{-1}
$$

By [35, Lemma 2.1(2)], we have

$$
\left\langle z, z^{*}\right\rangle=0, \quad \forall\left(z, z^{*}\right) \in \operatorname{gra}\left(-A^{*}\right) \cap X \times X^{*} .
$$

Hence $\left.A^{*}\right|_{X}$ is skew. Let $\left(x, x^{*}\right) \in X \times X^{*}$. Then by (17), we have

$$
\begin{aligned}
F_{A}\left(x, x^{*}\right) & =\sup _{\left(a, a^{*}\right) \in \operatorname{gra} A}\left\{\left\langle x, a^{*}\right\rangle+\left\langle x^{*}, a\right\rangle-\left\langle a, a^{*}\right\rangle\right\} \\
& \geq \sup _{\left(a, a^{*}\right) \in \operatorname{gra}\left(-A^{*}\right) \cap X \times X^{*}}\left\{\left\langle x, a^{*}\right\rangle+\left\langle x^{*}, a\right\rangle-\left\langle a, a^{*}\right\rangle\right\} \\
& =\sup _{\left(a, a^{*}\right) \in \operatorname{gra}\left(-A^{*}\right) \cap X \times X^{*}}\left\{\left\langle x, a^{*}\right\rangle+\left\langle x^{*}, a\right\rangle\right\} \\
& =\iota\left(\operatorname{gra}\left(-A^{*}\right) \cap X \times X^{*}\right)^{\perp} \cap X^{*} \times X \\
& =\iota_{\operatorname{gra} A}\left(x, x^{*}\right) \quad(\text { by (16) }) .
\end{aligned}
$$

Hence by Fact 3.5

$$
F_{A}\left(x, x^{*}\right)=\left\langle x, x^{*}\right\rangle+\iota_{\operatorname{gra} A}\left(x, x^{*}\right) .
$$

Hence by Corollary 3.24, $A$ is non-enlargeable.

The following corollary, which holds in a general Banach space, provides a characterization of non-enlargeable operators under a closedness assumption on the graph. A characterization of non-enlargeable linear operators for reflexive spaces (in which the closure assumption is hidden) was established by Svaiter in [35, Theorem 2.5]. 
Corollary 4.2 Let $A: X \rightrightarrows X^{*}$ be maximally monotone and suppose that gra $A$ is weak $\times$ weak $k^{*}$ closed. Select $\left(a, a^{*}\right) \in \operatorname{gra} A$ and set gra $\widetilde{A}:=\operatorname{gra} A-\left\{\left(a, a^{*}\right)\right\}$. Then $A$ is non-enlargeable if and only if gra $A$ is convex and $\operatorname{gra}\left(-\widetilde{A}^{*}\right) \cap X \times X^{*} \subseteq$ gra $\widetilde{A}$. In particular, $\left\langle x, x^{*}\right\rangle=0, \forall\left(x, x^{*}\right) \in \operatorname{gra} \widetilde{A}^{*} \cap X \times X^{*}$.

Proof. " $\Rightarrow$ ": By the assumption that $A$ is non-enlargeable, so is $\widetilde{A}$. By Fact 3.22 , gra $A$ is convex and then gra $A$ is affine by Fact 3.12 . Thus $\widetilde{A}$ is a linear relation. Now we can apply Theorem 4.1 to $\widetilde{A}$. " $\Leftarrow$ ": Apply Fact 3.12 and Theorem 4.1 directly.

Remark 4.3 We cannot remove the condition that "gra $A$ is convex" in Corollary 4.2, For example, let $X=\mathbb{R}^{n}$ with the Euclidean norm. Suppose that $f:=\|\cdot\|$. Then $\partial f$ is maximally monotone by Fact 3.3 , and hence gra $\partial f$ is weak $\times$ weak $^{*}$ closed. Now we show that

$$
\operatorname{gra}(\partial f)^{*}=\{(0,0)\}
$$

Note that

$$
\partial f(x)= \begin{cases}B_{X}, & \text { if } x=0 \\ \left\{\frac{x}{\|x\|}\right\}, & \text { otherwise }\end{cases}
$$

Let $\left(z, z^{*}\right) \in \operatorname{gra}(\partial f)^{*}$. By (21), we have $\left(0, B_{X}\right) \subseteq \operatorname{gra} \partial f$ and thus

$$
\left\langle-z, B_{X}\right\rangle=0 .
$$

Thus $z=0$. Hence

$$
\left\langle z^{*}, a\right\rangle=0, \quad \forall a \in \operatorname{dom} \partial f .
$$

Since $\operatorname{dom} \partial f=X, z^{*}=0$ by (23). Hence $\left(z, z^{*}\right)=(0,0)$ and thus (20) holds. By (20), gra $-(\partial f)^{*} \subseteq$ gra $\partial f$. However, gra $\partial f$ is not convex. Indeed, let $e_{k}=(0, \ldots, 0,1,0, \cdots, 0)$ : the $k$ th entry is 1 and the others are 0 . Take

$$
a=\frac{e_{1}-e_{2}}{\sqrt{2}} \quad \text { and } \quad b=\frac{e_{2}-e_{3}}{\sqrt{2}} .
$$

Then $(a, a) \in$ gra $\partial f$ and $(b, b) \in$ gra $\partial f$ by (21), but

$$
\frac{1}{2}(a, a)+\frac{1}{2}(b, b) \notin \operatorname{gra} \partial f .
$$

Hence $\partial f$ is enlargeable by Fact 3.22 .

In the case of a skew operator we can be more exacting: 
Corollary 4.4 Let $A: X \rightrightarrows X^{*}$ be a maximally monotone and skew operator and $\varepsilon \geq 0$. Then

(i) $\operatorname{gra} A_{\varepsilon}=\left\{\left(x, x^{*}\right) \in \operatorname{gra}\left(-A^{*}\right) \cap X \times X^{*} \mid\left\langle x, x^{*}\right\rangle \geq-\varepsilon\right\}$.

(ii) $A$ is non-enlargeable if and only if $\operatorname{gra} A=\operatorname{gra}\left(-A^{*}\right) \cap X \times X^{*}$.

(iii) $A$ is non-enlargeable if and only if $\operatorname{dom} A=\operatorname{dom} A^{*} \cap X$.

(iv) Assume that $X$ is reflexive. Then $F_{A^{*}}=\iota_{\mathrm{gra}} A^{*}+\langle\cdot, \cdot\rangle$ and hence $A^{*}$ is non-enlargeable.

Proof. (i); By [4, Lemma 3.1], we have

$$
F_{A}=\iota_{\operatorname{gra}\left(-A^{*}\right) \cap X \times X^{*}} .
$$

Hence $\left(x, x^{*}\right) \in \operatorname{gra} A_{\varepsilon}$ if and only if $F_{A}\left(x, x^{*}\right) \leq\left\langle x, x^{*}\right\rangle+\varepsilon$. This yields $\left(x, x^{*}\right) \in \operatorname{gra}\left(-A^{*}\right) \cap$ $X \times X^{*}$ and $0 \leq\left\langle x, x^{*}\right\rangle+\varepsilon$.

(ii) From Fact 3.22 we have that $\operatorname{dom} F_{A}=\operatorname{gra} A$. The claim now follows by combining the latter with (24).

(iii), For " $\Rightarrow$ ": use (ii), " $\Leftarrow$ ": Since $A$ is skew, we have gra $\left(-A^{*}\right) \cap X \times X^{*} \supseteq$ gra $A$. Using this and (ii), it suffices to show that $\operatorname{gra}\left(-A^{*}\right) \cap X \times X^{*} \subseteq \operatorname{gra} A$. Let $\left(x, x^{*}\right) \in \operatorname{gra}\left(-A^{*}\right) \cap$ $X \times X^{*}$. By the assumption, $x \in \operatorname{dom} A$. Let $y^{*} \in A x$. Note that $\left\langle x,-x^{*}\right\rangle=\left\langle x, y^{*}\right\rangle=0$, where the first equality follows from the definition of $A^{*}$ and the second one from the fact that $A$ is skew. In this case we claim that $\left(x, x^{*}\right)$ is monotonically related to gra $A$. Indeed, let $\left(a, a^{*}\right) \in \operatorname{gra} A$. Since $A$ is skew we have $\left\langle a, a^{*}\right\rangle=0$. Thus

$$
\left\langle x-a, x^{*}-a^{*}\right\rangle=\left\langle x, x^{*}\right\rangle-\left\langle\left(x^{*}, x\right),\left(a, a^{*}\right)\right\rangle+\left\langle a, a^{*}\right\rangle=0
$$

since $\left(x^{*}, x\right) \in(\operatorname{gra} A)^{\perp}$ and $\left\langle x, x^{*}\right\rangle=\left\langle a, a^{*}\right\rangle=0$. Hence $\left(x, x^{*}\right)$ is monotonically related to gra $A$. By maximality we conclude $\left(x, x^{*}\right) \in \operatorname{gra} A$. Hence gra $\left(-A^{*}\right) \cap X \times X^{*} \subseteq$ gra $A$.

[iv) Now assume that $X$ is reflexive. By [16, Theorem 2] (or see [39, 33]), $A^{*}$ is maximally monotone. Since gra $A \subseteq \operatorname{gra}\left(-A^{*}\right)$ we deduce that $\operatorname{gra}\left(-A^{* *}\right)=\operatorname{gra}(-A) \subseteq \operatorname{gra} A^{*}$. The latter inclusion and Theorem 4.1 applied to the operator $A^{*}$ yields $A^{*}$ non-enlargeable. The conclusion now follows by applying Corollary 3.24 to $A^{*}$.

\subsection{Limiting examples and remarks}

It is possible for a non-enlargeable maximally monotone operator to be non-skew. This is the case for the operator $A^{*}$ in Example 4.7. 
Example 4.5 Let $A: X \rightrightarrows X^{*}$ be a non-enlargeable maximally monotone operator. By Fact 3.22 and Fact [3.12, gra $A$ is affine. Let $f: X \rightarrow]-\infty,+\infty]$ be a proper lower semicontinuous convex function with $\operatorname{dom} A \cap \operatorname{int} \operatorname{dom} \partial f \neq \varnothing$ such that $\operatorname{dom} A \cap \operatorname{dom} \partial f$ is not an affine set. By Fact 3.10, $A+\partial f$ is maximally monotone. Since gra $(A+\partial f)$ is not affine, $A+\partial f$ is enlargeable.

The operator in the following example was studied in detail in [11].

Fact 4.6 Suppose that $X=\ell^{2}$, and that $A: \ell^{2} \rightrightarrows \ell^{2}$ is given by

$$
A x:=\frac{\left(\sum_{i<n} x_{i}-\sum_{i>n} x_{i}\right)_{n \in \mathbb{N}}}{2}=\left(\sum_{i<n} x_{i}+\frac{1}{2} x_{n}\right)_{n \in \mathbb{N}}, \quad \forall x=\left(x_{n}\right)_{n \in \mathbb{N}} \in \operatorname{dom} A,
$$

where $\operatorname{dom} A:=\left\{x:=\left(x_{n}\right)_{n \in \mathbb{N}} \in \ell^{2} \mid \sum_{i \geq 1} x_{i}=0,\left(\sum_{i \leq n} x_{i}\right)_{n \in \mathbb{N}} \in \ell^{2}\right\}$ and $\sum_{i<1} x_{i}:=0$. Now [11, Propositions 3.6] states that

$$
A^{*} x=\left(\frac{1}{2} x_{n}+\sum_{i>n} x_{i}\right)_{n \in \mathbb{N}}
$$

where

$$
x=\left(x_{n}\right)_{n \in \mathbb{N}} \in \operatorname{dom} A^{*}=\left\{x=\left(x_{n}\right)_{n \in \mathbb{N}} \in \ell^{2} \mid\left(\sum_{i>n} x_{i}\right)_{n \in \mathbb{N}} \in \ell^{2}\right\} .
$$

Then $A$ is an at most single-valued linear relation such that the following hold (proofs of all claims are in brackets).

(i) A is maximally monotone and skew ([11, Propositions 3.5 and 3.2]).

(ii) $A^{*}$ is maximally monotone but not skew ([11, Theorem 3.9 and Proposition 3.6]).

(iii) $\operatorname{dom} A$ is dense in $\ell^{2}$ ([26, Theorem 2.5]), and $\operatorname{dom} A \varsubsetneqq \operatorname{dom} A^{*}$ ([11, Proposition 3.6]).

(iv) $\left\langle A^{*} x, x\right\rangle=\frac{1}{2} s^{2}, \quad \forall x=\left(x_{n}\right)_{n \in \mathbb{N}} \in \operatorname{dom} A^{*}$ with $s:=\sum_{i \geq 1} x_{i}$ ([11, Proposition 3.7]).

Example 4.7 Suppose that $X$ and $A$ are as in Fact 4.6. Then $A$ is enlargeable but $A^{*}$ is non-enlargeable and is not skew. Moreover,

$$
\operatorname{gra} A_{\varepsilon}=\left\{\left(x, x^{*}\right) \in \operatorname{gra}\left(-A^{*}\right)|| \sum_{i \geq 1} x_{i} \mid \leq \sqrt{2 \varepsilon}, x=\left(x_{n}\right)_{n \in \mathbb{N}}\right\},
$$

where $\varepsilon \geq 0$. 
Proof. By Corollary 4.4(iii) and Fact 4.6](iii), $A$ must be enlargeable. For the second claim, note that $X=\ell^{2}$ is reflexive, and hence by Fact 4.6)(i) and Corollary 4.4)(iv), for every skew operator we must have $A^{*}$ non-enlargeable. For the last statement, apply Corollary 4.4)(i) and Fact 4.6](iv) directly to obtain gra $A_{\varepsilon}$.

Example 4.8 Let $C$ be a nonempty closed convex subset of $X$ and $\varepsilon \geq 0$. Then

$$
\operatorname{gra}\left(N_{C}\right)_{\varepsilon}=\left\{\left(x, x^{*}\right) \in C \times X^{*} \mid \sigma_{C}\left(x^{*}\right) \leq\left\langle x, x^{*}\right\rangle+\varepsilon\right\} .
$$

Proof. By Fact 3.19, we have

$$
\begin{aligned}
\left(x, x^{*}\right) \in \operatorname{gra}\left(N_{C}\right)_{\varepsilon} & \Leftrightarrow F_{N_{C}}\left(x, x^{*}\right)=\iota_{C}(x)+\sigma_{C}\left(x^{*}\right) \leq\left\langle x, x^{*}\right\rangle+\varepsilon \\
& \Leftrightarrow x \in C, \sigma_{C}\left(x^{*}\right) \leq\left\langle x, x^{*}\right\rangle+\varepsilon .
\end{aligned}
$$

Example 4.9 Let $f(x):=\|x\|, \forall x \in X$ and $\varepsilon \geq 0$. Then

$$
\operatorname{gra}(\partial f)_{\varepsilon}=\left\{\left(x, x^{*}\right) \in X \times B_{X^{*}} \mid\|x\| \leq\left\langle x, x^{*}\right\rangle+\varepsilon\right\} .
$$

In particular, $(\partial f)_{\varepsilon}(0)=B_{X^{*}}$.

Proof. Note that $f$ is sublinear, and hence by Fact 3.20 and Remark 3.21 we can write

$$
\begin{aligned}
\left(x, x^{*}\right) \in \operatorname{gra}(\partial f)_{\varepsilon} & \Leftrightarrow F_{\partial f}\left(x, x^{*}\right)=f(x)+f^{*}\left(x^{*}\right) \leq\left\langle x, x^{*}\right\rangle+\varepsilon \quad(\text { by (13) }) \\
& \Leftrightarrow\|x\|+\iota_{B_{X^{*}}}\left(x^{*}\right) \leq\left\langle x, x^{*}\right\rangle+\varepsilon \quad \text { (by [41, Corollary 2.4.16]) } \\
& \Leftrightarrow x^{*} \in B_{X^{*}},\|x\| \leq\left\langle x, x^{*}\right\rangle+\varepsilon .
\end{aligned}
$$

Hence $(\partial f)_{\varepsilon}(0)=B_{X^{*}}$.

Example 4.10 Let $p>1$ and $f(x):=\frac{1}{p}\|x\|^{p}, \forall x \in X$. Then

$$
(\partial f)_{\varepsilon}(0)=p^{\frac{1}{p}}(q \varepsilon)^{\frac{1}{q}} B_{X^{*}}
$$

where $\frac{1}{p}+\frac{1}{q}=1$ and $\varepsilon \geq 0$. 
Proof. We have

$$
\begin{aligned}
x^{*} \in(\partial f)_{\varepsilon}(0) & \Leftrightarrow\left\langle x^{*}-y^{*},-y\right\rangle \geq-\varepsilon, \quad \forall y^{*} \in \partial f(y) \\
& \Leftrightarrow\left\langle x^{*},-y\right\rangle+\|y\|^{p} \geq-\varepsilon, \quad \forall y \in X \\
& \Leftrightarrow\left\langle x^{*}, y\right\rangle-\|y\|^{p} \leq \varepsilon, \quad \forall y \in X \\
& \Leftrightarrow p \sup _{y \in X}\left[\left\langle\frac{1}{p} x^{*}, y\right\rangle-\frac{1}{p}\|y\|^{p}\right] \leq \varepsilon \\
& \Leftrightarrow p \cdot \frac{1}{q}\left\|\frac{1}{p} x^{*}\right\|^{q} \leq \varepsilon \\
& \Leftrightarrow\left\|x^{*}\right\|^{q} \leq q \varepsilon p^{q-1}=q \varepsilon p^{\frac{q}{p}} \\
& \Leftrightarrow x^{*} \in p^{\frac{1}{p}}(q \varepsilon)^{\frac{1}{q}} B_{X^{*}} .
\end{aligned}
$$

\subsection{Applications of Fitzpatrick's last function}

For a monotone linear operator $A: X \rightarrow X^{*}$ it will be very useful to define the following quadratic function (which is actually a special case of Fitzpatrick's last function [15] for the linear relation $A)$ :

$$
q_{A}: x \mapsto \frac{1}{2}\langle x, A x\rangle .
$$

Then $q_{A}=q_{A_{+}}$. We shall use the well known fact (see, e.g., [26]) that

$$
\nabla q_{A}=A_{+},
$$

where the gradient operator $\nabla$ is understood in the Gâteaux sense.

The next result was first given in [9, Proposition 2.2] for a reflexive space. The proof is easily adapted to a general Banach space.

Fact 4.11 Let $A: X \rightarrow X^{*}$ be linear continuous, symmetric and monotone. Then

$$
\left(\forall\left(x, x^{*}\right) \in X \times X^{*}\right) \quad q_{A}^{*}\left(x^{*}+A x\right)=q_{A}(x)+\left\langle x, x^{*}\right\rangle+q_{A}^{*}\left(x^{*}\right)
$$

and $q_{A}^{*} \circ A=q_{A}$.

The next result was first proven in [3, Proposition 2.2(v)] in Hilbert space. We now extend it to a general Banach space.

Proposition 4.12 Let $A: X \rightarrow X^{*}$ be linear and monotone. Then

$$
F_{A}\left(x, x^{*}\right)=2 q_{A_{+}}^{*}\left(\frac{1}{2} x^{*}+\frac{1}{2} A^{*} x\right)=\frac{1}{2} q_{A_{+}}^{*}\left(x^{*}+A^{*} x\right), \quad \forall\left(x, x^{*}\right) \in X \times X,
$$


and $\operatorname{ran} A_{+} \subseteq \operatorname{dom} \partial q_{A_{+}}^{*} \subseteq \operatorname{dom} q_{A_{+}}^{*} \subseteq \overline{\operatorname{ran} A_{+}}$. If ran $A_{+}$is closed, then $\operatorname{dom} q_{A_{+}}^{*}=$ $\operatorname{dom} \partial q_{A_{+}}^{*}=\operatorname{ran} A_{+}$.

Proof. By Fact 3.11, $\operatorname{dom} A^{*} \cap X=X$, so for every $x, y \in X$ we have $x, y \in \operatorname{dom} A^{*} \cap \operatorname{dom} A$. The latter fact and the definition of $A^{*}$ yield $\left\langle y, A^{*} x\right\rangle=\langle x, A y\rangle$. Hence for every $\left(x, x^{*}\right) \in$ $X \times X^{*}$,

$$
\begin{aligned}
F_{A}\left(x, x^{*}\right) & =\sup _{y \in X}\langle x, A y\rangle+\left\langle y, x^{*}\right\rangle-\langle y, A y\rangle \\
& =2 \sup _{y \in X}\left\langle y, \frac{1}{2} x^{*}+\frac{1}{2} A^{*} x\right\rangle-q_{A_{+}}(y) \\
& =2 q_{A_{+}}^{*}\left(\frac{1}{2} x^{*}+\frac{1}{2} A^{*} x\right) \\
& =\frac{1}{2} q_{A_{+}}^{*}\left(x^{*}+A^{*} x\right),
\end{aligned}
$$

where we also used the fact that $q_{A}=q_{A_{+}}$in the second equality. The third equality follows from the definition of Fenchel conjugate. By [41, Proposition 2.4.4(iv)],

$$
\operatorname{ran} \partial q_{A_{+}} \subseteq \operatorname{dom} \partial q_{A_{+}}^{*}
$$

By (27), $\operatorname{ran} \partial q_{A_{+}}=\operatorname{ran} A_{+}$. Then by (31),

$$
\operatorname{ran} A_{+} \subseteq \operatorname{dom} \partial q_{A_{+}}^{*} \subseteq \operatorname{dom} q_{A_{+}}^{*}
$$

Then by the Brøndsted-Rockafellar Theorem (see [41, Theorem 3.1.2]),

$$
\operatorname{ran} A_{+} \subseteq \operatorname{dom} \partial q_{A_{+}}^{*} \subseteq \operatorname{dom} q_{A_{+}}^{*} \subseteq \overline{\operatorname{ran} A_{+}}
$$

Hence, under the assumption that $\operatorname{ran} A_{+}$is closed, we have $\operatorname{ran} A_{+}=\operatorname{dom} \partial q_{A_{+}}^{*}=\operatorname{dom} q_{A_{+}}^{*}$.

We can now apply the last proposition to obtain a formula for the enlargement of a single valued-operator.

Proposition 4.13 (Enlargement of a monotone linear operator) Let $A: X \rightarrow X^{*}$ be a linear and monotone operator, and $\varepsilon \geq 0$. Then

$$
A_{\varepsilon}(x)=\left\{A x+z^{*} \mid q_{A}^{*}\left(z^{*}\right) \leq 2 \varepsilon\right\}, \quad \forall x \in X .
$$

Moreover, $A$ is non-enlargeable if and only if $A$ is skew.

Proof. Fix $x \in X, z^{*} \in X^{*}$ and $x^{*}=A x+z^{*}$. Then by Proposition 4.12 and Fact 4.11 ,

$$
\begin{aligned}
& x^{*} \in A_{\varepsilon}(x) \Leftrightarrow F_{A}\left(x, A x+z^{*}\right) \leq\left\langle x, A x+z^{*}\right\rangle+\varepsilon \\
& \Leftrightarrow \frac{1}{2} q_{A_{+}}^{*}\left(A x+z^{*}+A^{*} x\right) \leq\left\langle x, A x+z^{*}\right\rangle+\varepsilon \\
& \Leftrightarrow \frac{1}{2} q_{A_{+}}^{*}\left(A_{+}(2 x)+z^{*}\right) \leq\left\langle x, A x+z^{*}\right\rangle+\varepsilon \\
& \Leftrightarrow \frac{1}{2}\left[q_{A_{+}}^{*}\left(z^{*}\right)+2\left\langle x, z^{*}\right\rangle+2\langle x, A x\rangle\right] \leq\left\langle x, A x+z^{*}\right\rangle+\varepsilon \\
& \Leftrightarrow q_{A}^{*}\left(z^{*}\right) \leq 2 \varepsilon,
\end{aligned}
$$


where we also used in the last equivalence the fact that $q_{A}=q_{A_{+}}$. Now we show the second statement. By Fact 3.11, dom $A^{*} \cap X=X$. Then by Theorem 4.1 and Corollary 4.4](iii), we have $A$ is non-enlargeable if and only if $A$ is skew.

A result similar to Corollary 4.14 below was proved in [18, Proposition 2.2] in reflexive space. Their proof still requires the constraint that $\operatorname{ran}\left(A+A^{*}\right)$ is closed.

Corollary 4.14 Let $A: X \rightarrow X^{*}$ be a linear continuous and monotone operator such that $\operatorname{ran}\left(A+A^{*}\right)$ is closed. Then

$$
A_{\varepsilon}(x)=\left\{A x+\left(A+A^{*}\right) z \mid q_{A}(z) \leq \frac{1}{2} \varepsilon\right\}, \quad \forall x \in X .
$$

Proof. Proposition 4.13 yields

$$
x^{*} \in A_{\varepsilon}(x) \Leftrightarrow x^{*}=A x+z^{*}, q_{A}^{*}\left(z^{*}\right) \leq 2 \varepsilon .
$$

In particular, $z^{*} \in \operatorname{dom} q_{A}^{*}$. Since $\operatorname{ran}\left(A_{+}\right)$is closed, Proposition 4.12 yields

$$
\operatorname{ran}\left(A_{+}\right)=\operatorname{ran}\left(A+A^{*}\right)=\operatorname{dom} q_{A_{+}}^{*}=\operatorname{dom} q_{A}^{*} .
$$

The above expression and the fact that $z^{*} \in \operatorname{dom} q_{A}^{*}$ implies that there exists $z \in X$ such that $z^{*}=\left(A+A^{*}\right) z$. Note also that (by Fact 4.11)

$$
q_{A}^{*}\left(z^{*}\right)=q_{A_{+}}^{*}\left(z^{*}\right)=q_{A_{+}}^{*}\left(A_{+}(2 z)\right)=q_{A_{+}}(2 z)=4 q_{A}(z),
$$

where we used Fact 4.11 in the last equality. Using this in (34) gives

$$
\begin{aligned}
x^{*} \in A_{\varepsilon}(x) & \Leftrightarrow x^{*}=A x+\left(A+A^{*}\right) z, 4 q_{A}(z) \leq 2 \varepsilon \\
& \Leftrightarrow x^{*}=A x+\left(A+A^{*}\right) z, q_{A}(z) \leq \frac{1}{2} \varepsilon
\end{aligned}
$$

establishing the claim.

We conclude the section with two examples.

Example 4.15 (Rotation) Assume that $X$ is the Euclidean plane $\mathbb{R}^{2}$, let $\theta \in\left[0, \frac{\pi}{2}\right]$, and set

$$
A:=\left(\begin{array}{cc}
\cos \theta & -\sin \theta \\
\sin \theta & \cos \theta
\end{array}\right)
$$

Then for every $(\varepsilon, x) \in \mathbb{R}_{+} \times \mathbb{R}^{2}$,

$$
A_{\varepsilon}(x)=\left\{A x+v \mid v \in 2 \sqrt{(\cos \theta) \varepsilon} B_{X}\right\} .
$$


Proof. We consider two cases.

Case 1: $\theta=\frac{\pi}{2}$.

Then $A$ is skew operator. By Corollary 4.4, $A_{\varepsilon}=A$ and hence (36) holds.

Case 2: $\theta \in\left[0, \frac{\pi}{2}[\right.$.

Let $x \in \mathbb{R}^{2}$. Note that $\frac{A+A^{*}}{2}=(\cos \theta) \operatorname{Id}, q_{A}=\frac{\cos \theta}{2}\|\cdot\|^{2}$. Then by Corollary 4.14,

$$
A_{\varepsilon}(x)=\left\{A x+2(\cos \theta) z \mid q_{A}(z)=\frac{\cos \theta}{2}\|z\|^{2} \leq \frac{1}{2} \varepsilon\right\} .
$$

Thus,

$$
A_{\varepsilon}(x)=\{A x+v \mid\|v\| \leq 2 \sqrt{(\cos \theta) \varepsilon}\}=\left\{A x+v \mid v \in 2 \sqrt{(\cos \theta) \varepsilon} B_{X}\right\} .
$$

Example 4.16 (Identity) Assume that $X$ is a Hilbert space, and $A:=\mathrm{Id}$. Let $\varepsilon \geq 0$. Then

$$
\operatorname{gra} A_{\varepsilon}=\left\{\left(x, x^{*}\right) \in X \times X \mid x^{*} \in x+2 \sqrt{\varepsilon} B_{X}\right\} .
$$

Proof. By [7, Example 3.10], we have

$$
\begin{aligned}
\left(x, x^{*}\right) \in \operatorname{gra} A_{\varepsilon} & \Leftrightarrow \frac{1}{4}\left\|x+x^{*}\right\|^{2} \leq\left\langle x, x^{*}\right\rangle+\varepsilon \\
& \Leftrightarrow \frac{1}{4}\left\|x-x^{*}\right\|^{2} \leq \varepsilon \\
& \Leftrightarrow\left\|x-x^{*}\right\| \leq 2 \sqrt{\varepsilon} \\
& \Leftrightarrow x^{*} \in x+2 \sqrt{\varepsilon} B_{X} .
\end{aligned}
$$

\section{Sums of operators}

The conclusion of the lemma below has been established for reflexive Banach spaces in [10, Lemma 5.8]. Our proof for a general Banach space assumes the operators to be of type (FPV) and follows closely that of [10, Lemma 5.8]. 
Lemma 5.1 Let $A, B: X \rightrightarrows X^{*}$ be maximally monotone of type (FPV), and suppose that $\bigcup_{\lambda>0} \lambda[\operatorname{dom} A-\operatorname{dom} B]$ is a closed subspace of $X$. Then we have

$$
\bigcup_{\lambda>0} \lambda[\operatorname{dom} A-\operatorname{dom} B]=\bigcup_{\lambda>0} \lambda\left[P_{X} \operatorname{dom} F_{A}-P_{X} \operatorname{dom} F_{B}\right] .
$$

Proof. By Fact 3.5 and Fact 3.6, we have

$$
\begin{aligned}
& \bigcup_{\lambda>0} \lambda[\operatorname{dom} A-\operatorname{dom} B] \subseteq \bigcup_{\lambda>0} \lambda\left[P_{X} \operatorname{dom} F_{A}-P_{X} \operatorname{dom} F_{B}\right] \subseteq \bigcup_{\lambda>0} \lambda[\overline{\operatorname{dom} A}-\overline{\operatorname{dom} B}] \\
& \subseteq \bigcup_{\lambda>0} \lambda\left[\overline{\operatorname{dom} A-\operatorname{dom} B] \subseteq \bigcup_{\lambda>0} \lambda[\operatorname{dom} A-\operatorname{dom} B]}\right. \\
& =\bigcup_{\lambda>0} \lambda[\operatorname{dom} A-\operatorname{dom} B] \quad \text { (by the assumption). }
\end{aligned}
$$

Corollary 5.2 Let $A, B: X \rightrightarrows X^{*}$ be maximally monotone linear relations, and suppose that $\operatorname{dom} A-\operatorname{dom} B$ is a closed subspace. Then

$$
[\operatorname{dom} A-\operatorname{dom} B]=\bigcup_{\lambda>0} \lambda\left[P_{X} \operatorname{dom} F_{A}-P_{X} \operatorname{dom} F_{B}\right] .
$$

Proof. Directly apply Fact 3.8 and Lemma 5.1 .

Corollary 5.3 Let $A: X \rightrightarrows X^{*}$ be a maximally monotone linear relation and let $C \subseteq X$ be a nonempty and closed convex set. Assume that $\bigcup_{\lambda>0} \lambda[\operatorname{dom} A-C]$ is a closed subspace. Then

$$
\bigcup_{\lambda>0} \lambda\left[P_{X} \operatorname{dom} F_{A}-P_{X} \operatorname{dom} F_{N_{C}}\right]=\bigcup_{\lambda>0} \lambda[\operatorname{dom} A-C] .
$$

Proof. Let $B=N_{C}$. Then apply directly Fact 3.8, Fact 3.9 and Lemma 5.1 .

Theorem 5.4 below was proved in [10, Theorem 5.10] for a reflexive space. We extend it to a general Banach space.

Theorem 5.4 (Fitzpatrick function of the sum) Let $A, B: X \rightrightarrows X^{*}$ be maximally monotone linear relations, and suppose that $\operatorname{dom} A-\operatorname{dom} B$ is closed. Then

$$
F_{A+B}=F_{A} \square_{2} F_{B}
$$

and the partial infimal convolution is exact everywhere. 
Proof. Let $\left(z, z^{*}\right) \in X \times X^{*}$. By Fact 3.18, it suffices to show that there exists $v^{*} \in X^{*}$ such that

$$
F_{A+B}\left(z, z^{*}\right) \geq F_{A}\left(z, z^{*}-v^{*}\right)+F_{B}\left(z, v^{*}\right) .
$$

If $\left(z, z^{*}\right) \notin \operatorname{dom} F_{A+B}$, clearly, (37) holds.

Now assume that $\left(z, z^{*}\right) \in \operatorname{dom} F_{A+B}$. Then

$$
\begin{aligned}
& F_{A+B}\left(z, z^{*}\right) \\
& =\sup _{\left\{x, x^{*}, y^{*}\right\}}\left[\left\langle x, z^{*}\right\rangle+\left\langle z, x^{*}\right\rangle-\left\langle x, x^{*}\right\rangle+\left\langle z-x, y^{*}\right\rangle-\iota_{\operatorname{gra} A}\left(x, x^{*}\right)-\iota_{\operatorname{gra} B}\left(x, y^{*}\right)\right] .
\end{aligned}
$$

Let $Y=X^{*}$ and define $\left.\left.F, K: X \times X^{*} \times Y \rightarrow\right]-\infty,+\infty\right]$ respectively by

$$
\begin{aligned}
& F:\left(x, x^{*}, y^{*}\right) \in X \times X^{*} \times Y \rightarrow\left\langle x, x^{*}\right\rangle+\iota_{\operatorname{gra} A}\left(x, x^{*}\right) \\
& K:\left(x, x^{*}, y^{*}\right) \in X \times X^{*} \times Y \rightarrow\left\langle x, y^{*}\right\rangle+\iota_{\operatorname{gra} B}\left(x, y^{*}\right)
\end{aligned}
$$

Then by (38),

$$
F_{A+B}\left(z, z^{*}\right)=(F+K)^{*}\left(z^{*}, z, z\right)
$$

By Fact 3.13 and the assumptions, $F$ and $K$ are proper lower semicontinuous and convex. The definitions of $F$ and $K$ yield

$$
\operatorname{dom} F-\operatorname{dom} K=[\operatorname{dom} A-\operatorname{dom} B] \times X^{*} \times Y \text {, which is a closed subspace. }
$$

Thus by Fact 3.4 and (39), there exists $\left(z_{0}^{*}, z_{0}^{* *}, z_{1}^{* *}\right) \in X^{*} \times X^{* *} \times Y^{*}$ such that

$$
\begin{aligned}
F_{A+B}\left(z, z^{*}\right) & =F^{*}\left(z^{*}-z_{0}^{*}, z-z_{0}^{* *}, z-z_{1}^{* *}\right)+K^{*}\left(z_{0}^{*}, z_{0}^{* *}, z_{1}^{* *}\right) \\
& =F^{*}\left(z^{*}-z_{0}^{*}, z, 0\right)+K^{*}\left(z_{0}^{*}, 0, z\right) \quad\left(\text { by }\left(z, z^{*}\right) \in \operatorname{dom} F_{A+B}\right) \\
& =F_{A}\left(z, z^{*}-z_{0}^{*}\right)+F_{B}\left(z, z_{0}^{*}\right) .
\end{aligned}
$$

Thus (37) holds by taking $v^{*}=z_{0}^{*}$ and hence $F_{A+B}=F_{A} \square_{2} F_{B}$.

The next result was first obtained by Voisei in [37] while Simons gave a different proof in [32, Theorem 46.3]. We are now in position to provide a third approach.

Theorem 5.5 Let $A, B: X \rightrightarrows X^{*}$ be maximally monotone linear relations, and suppose that $\operatorname{dom} A-\operatorname{dom} B$ is closed. Then $A+B$ is maximally monotone.

Proof. By Fact 3.5, we have that $F_{A} \geq\langle\cdot, \cdot\rangle$ and $F_{B} \geq\langle\cdot, \cdot\rangle$. Using now Theorem 5.4 and (9) implies that $F_{A+B} \geq\langle\cdot, \cdot\rangle$. Combining the last inequality with Corollary 5.2 and Fact 3.14, we conclude that $A+B$ is maximally monotone. 
Theorem 5.6 Let $A, B: X \rightrightarrows X^{*}$ be maximally monotone linear relations, and suppose that $\operatorname{dom} A-\operatorname{dom} B$ is closed. Assume that $A$ and $B$ are non-enlargeable. Then

$$
F_{A+B}=\iota_{\operatorname{gra}(A+B)}+\langle\cdot, \cdot\rangle
$$

and hence $A+B$ is non-enlargeable.

Proof. By Corollary 3.24, we have

$$
F_{A}=\iota_{\operatorname{gra} A}+\langle\cdot, \cdot\rangle \quad \text { and } \quad F_{B}=\iota_{\operatorname{gra} B}+\langle\cdot, \cdot\rangle .
$$

Let $\left(x, x^{*}\right) \in X \times X^{*}$. Then by (40) and Theorem 5.4, we have

$$
\begin{aligned}
F_{A+B}\left(x, x^{*}\right) & =\min _{y^{*} \in X^{*}}\left\{\iota_{\operatorname{gra} A}\left(x, x^{*}-y^{*}\right)+\left\langle x^{*}-y^{*}, x\right\rangle+\iota_{\operatorname{gra} B}\left(x, y^{*}\right)+\left\langle y^{*}, x\right\rangle\right\} \\
& =\iota_{\operatorname{gra}(A+B)}\left(x, x^{*}\right)+\left\langle x^{*}, x\right\rangle .
\end{aligned}
$$

By Theorem 5.5 we have that $A+B$ is maximally monotone. Now we can apply Corollary 3.24 to $A+B$ to conclude that $A+B$ is non-enlargeable.

The proof of Theorem 5.7 in part follows that of [8, Theorem 3.1].

Theorem 5.7 Let $A: X \rightrightarrows X^{*}$ be a maximally monotone linear relation. Suppose $C$ is a nonempty closed convex subset of $X$, and that $\operatorname{dom} A \cap \operatorname{int} C \neq \varnothing$. Then $F_{A+N_{C}}=F_{A} \square_{2} F_{N_{C}}$, and the partial infimal convolution is exact everywhere.

Proof. Let $\left(z, z^{*}\right) \in X \times X^{*}$. By Fact 3.18, it suffices to show that there exists $v^{*} \in X^{*}$ such that

$$
F_{A+N_{C}}\left(z, z^{*}\right) \geq F_{A}\left(z, v^{*}\right)+F_{N_{C}}\left(z, z^{*}-v^{*}\right) .
$$

If $\left(z, z^{*}\right) \notin \operatorname{dom} F_{A+N_{C}}$, clearly, (41) holds.

Now assume that

$$
\left(z, z^{*}\right) \in \operatorname{dom} F_{A+N_{C}} \text {. }
$$

By Fact 3.10 and Fact 3.6,

$$
P_{X}\left[\operatorname{dom} F_{A+N_{C}}\right] \subseteq \overline{\left[\operatorname{dom}\left(A+N_{C}\right)\right]} \subseteq C .
$$

Thus, by (42), we have

$$
z \in C
$$


Set

$$
\left.\left.g: X \times X^{*} \rightarrow\right]-\infty,+\infty\right]:\left(x, x^{*}\right) \mapsto\left\langle x, x^{*}\right\rangle+\iota_{\operatorname{gra} A}\left(x, x^{*}\right)
$$

By Fact $3.13, g$ is convex. Hence,

$$
h=g+\iota_{C \times X^{*}}
$$

is convex as well. Let

$$
c_{0} \in \operatorname{dom} A \cap \operatorname{int} C
$$

and let $c_{0}^{*} \in A c_{0}$. Then $\left(c_{0}, c_{0}^{*}\right) \in \operatorname{gra} A \cap\left(\operatorname{int} C \times X^{*}\right)=\operatorname{dom} g \cap \operatorname{int} \operatorname{dom} \iota_{C \times X^{*}}$. Let us compute $F_{A+N_{C}}\left(z, z^{*}\right)$. As in (38) we can write

$$
\begin{aligned}
& F_{A+N_{C}}\left(z, z^{*}\right) \\
& =\sup _{\left(x, x^{*}, c^{*}\right)}\left[\left\langle x, z^{*}\right\rangle+\left\langle z, x^{*}\right\rangle-\left\langle x, x^{*}\right\rangle+\left\langle z-x, c^{*}\right\rangle-\iota_{\operatorname{gra}} A\left(x, x^{*}\right)-\iota_{\text {gra } N_{C}}\left(x, c^{*}\right)\right] \\
& \geq \sup _{\left(x, x^{*}\right)}\left[\left\langle x, z^{*}\right\rangle+\left\langle z, x^{*}\right\rangle-\left\langle x, x^{*}\right\rangle-\iota_{\operatorname{gra} A}\left(x, x^{*}\right)-\iota_{C \times X^{*}}\left(x, x^{*}\right)\right] \\
& =\sup _{\left(x, x^{*}\right)}\left[\left\langle x, z^{*}\right\rangle+\left\langle z, x^{*}\right\rangle-h\left(x, x^{*}\right)\right] \\
& =h^{*}\left(z^{*}, z\right),
\end{aligned}
$$

where we took $c^{*}=0$ in the inequality. By Fact 3.1. $\iota_{C \times X^{*}}$ is continuous at $\left(c_{0}, c_{0}^{*}\right) \in$ int dom $\iota_{C \times X^{*}}$. Since $\left(c_{0}, c_{0}^{*}\right) \in \operatorname{dom} g \cap \operatorname{int} \operatorname{dom} \iota_{C \times X^{*}}$ we can use Fact 3.2 to conclude the existence of $\left(y^{*}, y^{* *}\right) \in X^{*} \times X^{* *}$ such that

$$
\begin{aligned}
h^{*}\left(z^{*}, z\right) & =g^{*}\left(y^{*}, y^{* *}\right)+\iota_{C \times X^{*}}^{*}\left(z^{*}-y^{*}, z-y^{* *}\right) \\
& =g^{*}\left(y^{*}, y^{* *}\right)+\iota_{C}^{*}\left(z^{*}-y^{*}\right)+\iota_{\{0\}}\left(z-y^{* *}\right) .
\end{aligned}
$$

Then by (42) and (47) we must have $z=y^{* *}$. Thus by (47) and the definition of $g$ we have

$$
\begin{aligned}
& F_{A+N_{C}}\left(z, z^{*}\right) \geq g^{*}\left(y^{*}, z\right)+\iota_{C}^{*}\left(z^{*}-y^{*}\right)=F_{A}\left(z, y^{*}\right)+\iota_{C}^{*}\left(z^{*}-y^{*}\right) \\
& =F_{A}\left(z, y^{*}\right)+\iota_{C}^{*}\left(z^{*}-y^{*}\right)+\iota_{C}(z) \quad(\text { by }(43)) \\
& =F_{A}\left(z, y^{*}\right)+F_{N_{C}}\left(z, z^{*}-y^{*}\right) \quad(\text { by Fact 3.19) }
\end{aligned}
$$

Hence (41) holds by taking $v^{*}=y^{*}$ and thus $F_{A+N_{C}}=F_{A} \square_{2} F_{N_{C}}$.

We decode the prior result as follows:

Corollary 5.8 (Normal cone) Let $A: X \rightrightarrows X^{*}$ be a maximally monotone linear relation. Suppose $C$ is a nonempty closed convex subset of $X$, and that $\operatorname{dom} A \cap \operatorname{int} C \neq \varnothing$. Then $A+N_{C}$ is maximally monotone. 
Proof. By Fact 3.5, we have that $F_{A} \geq\langle\cdot, \cdot\rangle$ and $F_{N_{C}} \geq\langle\cdot, \cdot\rangle$. Using now Theorem 5.7 and (9) implies that $F_{A+N_{C}} \geq\langle\cdot, \cdot\rangle$. Combining the last inequality with Corollary 5.2 and Fact 3.14, we conclude that $A+N_{C}$ is maximally monotone.

To conclude we revisit a quite subtle example. All statements in the fact below have been proved in [4, Example 4.1 and Theorem 3.6(vii)].

Fact 5.9 Consider $X:=c_{0}$, with norm $\|\cdot\|_{\infty}$ so that $X^{*}=\ell^{1}$ with norm $\|\cdot\|_{1}$, and $X^{* *}=\ell^{\infty}$ with second dual norm $\|\cdot\|_{*}$. Fix $\alpha:=\left(\alpha_{n}\right)_{n \in \mathbb{N}} \in \ell^{\infty}$ with $\lim \sup \alpha_{n} \neq 0$, and define $A_{\alpha}: \ell^{1} \rightarrow \ell^{\infty}$ by

$$
\left(A_{\alpha} x^{*}\right)_{n}:=\alpha_{n}^{2} x_{n}^{*}+2 \sum_{i>n} \alpha_{n} \alpha_{i} x_{i}^{*}, \quad \forall x^{*}=\left(x_{n}^{*}\right)_{n \in \mathbb{N}} \in \ell^{1} .
$$

Finally, let $T_{\alpha}: c_{0} \rightrightarrows X^{*}$ be defined by

$$
\begin{aligned}
\operatorname{gra} T_{\alpha} & :=\left\{\left(-A_{\alpha} x^{*}, x^{*}\right) \mid x^{*} \in X^{*},\left\langle\alpha, x^{*}\right\rangle=0\right\} \\
& =\left\{\left(\left(-\sum_{i>n} \alpha_{n} \alpha_{i} x_{i}^{*}+\sum_{i<n} \alpha_{n} \alpha_{i} x_{i}^{*}\right)_{n}, x^{*}\right) \mid x^{*} \in X^{*},\left\langle\alpha, x^{*}\right\rangle=0\right\} .
\end{aligned}
$$

Then

(i) $\left\langle A_{\alpha} x^{*}, x^{*}\right\rangle=\left\langle\alpha, x^{*}\right\rangle^{2}, \quad \forall x^{*}=\left(x_{n}^{*}\right)_{n \in \mathbb{N}} \in \ell^{1}$ and so (49) is well defined.

(ii) $A_{\alpha}$ is a maximally monotone operator on $\ell^{1}$.

(iii) $T_{\alpha}$ is a maximally monotone and skew operator on $c_{0}$.

(iv) $F_{T_{\alpha}}=\iota_{C}$, where $C:=\left\{\left(-A_{\alpha} x^{*}, x^{*}\right) \mid x^{*} \in X^{*}\right\}$.

This set of affairs allows us to show the following:

Example 5.10 Let $X=c_{0}, A_{\alpha}, C$, and $T_{\alpha}$ be defined as in Fact 5.9, Then $T_{\alpha}: c_{0} \rightrightarrows \ell^{1}$ is a maximally monotone enlargeable skew linear relation. Indeed

$$
\operatorname{gra}\left(T_{\alpha}+N_{B_{X}}\right)_{\varepsilon}=\left\{\left(-A_{\alpha} x^{*}, z^{*}\right) \in B_{X} \times X^{*} \mid x^{*} \in X,\left\|z^{*}-x^{*}\right\|_{1} \leq\left\langle-A_{\alpha} x^{*}, z^{*}\right\rangle+\varepsilon\right\} .
$$

Proof. From (49), we have that gra $T_{\alpha} \varsubsetneqq C$ therefore Fact 5.9$]$ (iv) yields $F_{T_{\alpha}} \neq \iota_{\text {gra } T_{\alpha}}+\langle\cdot, \cdot\rangle$. Using now Fact 5.9](iii) and Corollary 3.24, we conclude that $T_{\alpha}$ is enlargeable.

Now we determine gra $\left(T_{\alpha}+N_{B_{X}}\right)_{\varepsilon}$. By Fact 5.9](iii), Theorem 5.7 and (4), we have

$$
\left(z, z^{*}\right) \in \operatorname{gra}\left(T_{\alpha}+N_{B_{X}}\right)_{\varepsilon}
$$




$$
\begin{aligned}
& \Leftrightarrow F_{T_{\alpha}} \square_{2} F_{N_{B_{X}}}\left(z, z^{*}\right) \leq\left\langle z, z^{*}\right\rangle+\varepsilon \\
& \Leftrightarrow F_{T_{\alpha}}\left(z, x^{*}\right)+\iota_{B_{X}}(z)+\iota_{B_{X}}^{*}\left(z^{*}-x^{*}\right) \leq\left\langle z, z^{*}\right\rangle+\varepsilon, \exists x^{*} \in X^{*} \quad \text { (by Fact 3.19) } \\
& \left.\Leftrightarrow z \in B_{X}, \iota_{C}\left(z, x^{*}\right)+\left\|z^{*}-x^{*}\right\|_{1} \leq\left\langle z, z^{*}\right\rangle+\varepsilon, \exists x^{*} \in X^{*} \text { (by Fact 5.9](iv) }\right) \\
& \Leftrightarrow z=-A_{\alpha} x^{*} \in B_{X},\left\|z^{*}-x^{*}\right\|_{1} \leq\left\langle z, z^{*}\right\rangle+\varepsilon, \exists x^{*} \in X^{*} \\
& \Leftrightarrow z=-A_{\alpha} x^{*} \in B_{X},\left\|z^{*}-x^{*}\right\|_{1} \leq\left\langle-A_{\alpha} x^{*}, z^{*}\right\rangle+\varepsilon, \exists x^{*} \in X^{*} .
\end{aligned}
$$

This is the desired result.

\section{References}

[1] H. Attouch and H. Brézis, "Duality for the sum of convex functions in general Banach spaces", Aspects of Mathematics and its Applications, J. A. Barroso, ed., Elsevier Science Publishers, pp. 125-133, 1986.

[2] S. Bartz, H.H. Bauschke, J.M. Borwein, S. Reich, and X. Wang, "Fitzpatrick functions, cyclic monotonicity and Rockafellar's antiderivative", Nonlinear Analysis, vol. 66, pp. 1198-1223, 2007.

[3] H.H. Bauschke, J.M. Borwein, and X. Wang, "Fitzpatrick functions and continuous linear monotone operators", SIAM Journal on Optimization, vol. 18, pp. 789-809, 2007.

[4] H.H. Bauschke, J.M. Borwein, X. Wang and L. Yao, "Construction of pathological maximally monotone operators on non-reflexive Banach spaces", submitted; http://arxiv.org/abs/1108.1463v1, August 2011.

[5] H.H. Bauschke, J.M. Borwein, X. Wang and L. Yao, "Monotone operators and "bigger conjugate" functions", submitted; http://arxiv.org/abs/1108.2578v1, August 2011.

[6] H.H. Bauschke and P.L. Combettes, Convex Analysis and Monotone Operator Theory in Hilbert Spaces, Springer-Verlag, 2011.

[7] H.H. Bauschke, D.A. McLaren, and H.S. Sendov, "Fitzpatrick functions: inequalities, examples and remarks on a problem by S. Fitzpatrick", Journal of Convex Analysis, vol. 13, pp. 499-523, 2006.

[8] H.H. Bauschke, X. Wang, and L. Yao, "An answer to S. Simons' question on the maximal monotonicity of the sum of a maximal monotone linear operator and a normal cone operator", Set-Valued and Variational Analysis, vol. 17, pp. 195-201, 2009.

[9] H.H. Bauschke, X. Wang, and L. Yao, "Autoconjugate representers for linear monotone operators", Mathematical Programming (Series B), vol. 123, pp. 5-24, 2010

[10] H.H. Bauschke, X. Wang, and L. Yao, "Monotone linear relations: maximality and Fitzpatrick functions", Journal of Convex Analysis, vol. 16, pp. 673-686, 2009. 
[11] H.H. Bauschke, X. Wang, and L. Yao, "Examples of discontinuous maximal monotone linear operators and the solution to a recent problem posed by B.F. Svaiter", Journal of Mathematical Analysis and Applications, vol. 370, pp. 224-241, 2010.

[12] J.M. Borwein, "Maximal monotonicity via convex analysis", Journal of Convex Analysis, vol. 13, pp. 561-586, 2006.

[13] J.M. Borwein, "Maximality of sums of two maximal monotone operators in general Banach space", Proceedings of the American Mathematical Society, vol. 135, pp. 3917-3924, 2007.

[14] J.M. Borwein, "Fifty years of maximal monotonicity", Optimization Letters, vol. 4, pp. 473490, 2010.

[15] J.M. Borwein and J.D. Vanderwerff, Convex Functions, Cambridge University Press, 2010.

[16] H. Brézis and F.E. Browder, "Linear maximal monotone operators and singular nonlinear integral equations of Hammerstein type", in Nonlinear Analysis (collection of papers in honor of Erich H. Rothe), Academic Press, pp. 31-42, 1978.

[17] A. Brøndsted and R. T. Rockafellar, "On the subdifferentiability of convex functions", Proceedings of the American Mathematical Society, vol. 16, pp. 605-611, 1965.

[18] R.S. Burachik and A.N. Iusem, On non-enlargeable and fully enlargeable monotone operators, Journal of Convex Analysis, vol. 13, pp. 603-622, 2006.

[19] R.S. Burachik and A.N. Iusem, Set-Valued Mappings and Enlargements of Monotone Operators, Springer-Verlag, 2008.

[20] R.S. Burachik, B.F. Svaiter, "Maximal monotone operators, convex functions and a special family of enlargements", Set-Valued Analysis, vol. 10, pp. 297-316, 2002.

[21] S. Fitzpatrick, "Representing monotone operators by convex functions", in Workshop/Miniconference on Functional Analysis and Optimization (Canberra 1988), Proceedings of the Centre for Mathematical Analysis, Australian National University, vol. 20, Canberra, Australia, pp. 59-65, 1988.

[22] S.P. Fitzpatrick and R.R. Phelps, "Some properties of maximal monotone operators on nonreflexive Banach spaces", Set-Valued Analysis, vol. 3, pp. 51-69, 1995.

[23] M. Marques Alves and B.F. Svaiter, "A new proof for maximal monotonicity of subdifferential operators", Journal of Convex Analysis, vol. 15, pp. 345-348, 2008.

[24] M. Marques Alves and B.F. Svaiter, "Maximal monotone operators with a unique extension to the bidual", Journal of Convex Analysis, vol. 16, pp. 409-421, 2009

[25] R.R. Phelps, Convex Functions, Monotone Operators and Differentiability, 2nd Edition, Springer-Verlag, 1993.

[26] R.R. Phelps and S. Simons, "Unbounded linear monotone operators on nonreflexive Banach spaces", Journal of Convex Analysis, vol. 5, pp. 303-328, 1998. 
[27] R.T. Rockafellar, "Extension of Fenchel's duality theorem for convex functions", Duke Mathematical Journal, vol. 33, pp. 81-89, 1966.

[28] R.T. Rockafellar, "On the maximal monotonicity of sums on nonlinear monotone operators", Trans. Amer. Math. Soc., vol. 149, pp. 75-88, 1970.

[29] R.T. Rockafellar, "On the maximal monotonicity of subdifferential mappings", Pacific Journal of Mathematics, vol. 33, pp. 209-216, 1970.

[30] R.T. Rockafellar and R.J-B Wets, Variational Analysis, 3rd Printing, Springer-Verlag, 2009.

[31] S. Simons, Minimax and Monotonicity, Springer-Verlag, 1998.

[32] S. Simons, From Hahn-Banach to Monotonicity, Springer-Verlag, 2008.

[33] S. Simons, "A Brézis-Browder theorem for SSDB spaces"; http://arxiv.org/abs/1004.4251v3, September 2010.

[34] B. F. Svaiter, "A family of enlargements of maximal monotone operators", Set-Valued Analysis, vol. 8, pp. 311-328, 2000.

[35] B.F. Svaiter, "Non-enlargeable operators and self-cancelling operators", Journal of Convex Analysis, vol. 17, pp. 309-320, 2010.

[36] A. Verona and M.E. Verona, "Regular maximal monotone operators", Set-Valued Analysis, vol. 6, pp. 303-312, 1998.

[37] M.D. Voisei, "The sum theorem for linear maximal monotone operators", Mathematical Sciences Research Journal, vol. 10, pp. 83-85, 2006.

[38] M.D. Voisei, "The sum and chain rules for maximal monotone operators", Set-Valued and Variational Analysis, vol. 16, pp. 461-476, 2008.

[39] L. Yao, "The Brézis-Browder Theorem revisited and properties of Fitzpatrick functions of order n", Fixed Point Theory for Inverse Problems in Science and Engineering (Banff 2009), Springer-Verlag, vol. 49, pp. 391-402, 2011.

[40] L. Yao, "The sum of a maximally monotone linear relation and the subdifferential of a proper lower semicontinuous convex function is maximally monotone", to appear Set-Valued and Variational Analysis.

[41] C. Zălinescu, Convex Analysis in General Vector Spaces, World Scientific Publishing, 2002.

[42] E. Zeidler, Nonlinear Functional Analysis and its Application, Vol II/B Nonlinear Monotone Operators, Springer-Verlag, New York-Berlin-Heidelberg, 1990. 
Acknowledgments. The authors thank Dr. Heinz Bauschke and Dr. Xianfu Wang for their valuable discussions and comments. Jonathan Borwein was partially supported by the Australian Research Council. The third author thanks CARMA at the University of Newcastle and the School of Mathematics and Statistics of University of South Australia for the support of his visit to Australia, which started this research. 